\title{
The Reliability Life of 7B04 Aluminum Alloy under Alternate Action of Corrosion and Fatigue
}

\author{
Changfan Li, Yuting He, Yu Feng, Liming Wu, and Sheng Zhang \\ Department of Aircraft Engineering, Aeronautics and Astronautics Engineering College, Air Force Engineering University, \\ Xian 710038, China \\ Correspondence should be addressed to Yuting He; heyut666@126.com
}

Received 19 January 2016; Accepted 15 March 2016

Academic Editor: Mitsuhiro Okayasu

Copyright (C) 2016 Changfan Li et al. This is an open access article distributed under the Creative Commons Attribution License, which permits unrestricted use, distribution, and reproduction in any medium, provided the original work is properly cited.

Experiment of alternate action of corrosion and fatigue on 7B04 aluminum alloy was carried out in this paper and fatigue life was obtained. One of the characteristics about fatigue life is that it is not always decreasing with the increasing of corrosion time. The surface splits caused by the corrosion of intruding and extruding slip steps on the surface and decreasing of stress concentration around corrosion pits were the main reasons. Single side allowance factor $k$ was proposed to calculate reliability fatigue life with both reliability and confidence. Reliability, confidence, and specimen number had affection on the value of $k$. The reliability influences more greatly the reliability fatigue life compared to the confidence. The safe fatigue life is smaller than median life and it was safe to administrate aircraft life using safe fatigue life.

\section{Introduction}

Service life is one of the most important indexes of aircraft structure. In order to ensure flight safety, the service life is actually a life limit with high reliability [1]. The determination of the life limit has been widely discussed to ensure that the aircraft is used safely and economically. Safe fatigue life is one main index of aircraft which is determined by fatigue test [2]. Meanwhile, the test data is obtained by fatigue experiments without corrosive environment.

Corrosion and fatigue are main damage to aircraft structure in service and many investigations have found the influence of corrosion and fatigue on the aircraft material [3-6]. Most studies focus on the corrosive environment and fatigue load simultaneously or separately. Precorrosion fatigue and corrosion fatigue damage rules have been broadly reported and indicate a synergism between corrosion and fatigue [7-9]. When the aircraft flies above $3 \mathrm{~km}$, the corrosive environment is minimal, for example, salt, temperature, moisture, and so on, while the fatigue load due to lift force, maneuver, flutter, and so forth is maximal. And when the aircraft is on the ground, the corrosion progress is maximal, while flight loads and vibration are not existent [10]. So fatigue progress and corrosion progress are alternate during the service progress of aircraft. Some research had been done to investigate the difference between alternate action of corrosion and fatigue and precorrosion fatigue. Menan and Henaff [11, 12] found the difference between alternate immersion corrosion and precorrosion in research of the affection of frequency and salt solution on crack growth of 2024 aluminum alloy. Chen et al. [13] showed that the fatigue life of $2 \mathrm{~A} 12$ aluminum alloy specimens in "fatigue + corrosion + fatigue" mode was longer than precorrosion specimens under the same corrosion condition. Jin and Yang [14] put forward a fatigue damage competitive rule. The experimental results under "fatigue + corrosion + fatigue" mode were in agreement with the rule. Fan [15] studied distribution law of the alternate action of corrosion and fatigue under "corrosion + fatigue + corrosion + fatigue $+\cdots$ " mode. Experimental results showed that it obeys the normal distribution. But these investigations failed to discuss the safe fatigue life and to predict the reliability fatigue life under the reliability and confidence.

As experiments of alternate action of fatigue and corrosion can account for aircraft service progress well, it is interesting to discuss the safe fatigue life under alternate state. In this study, the Al 7B04 alloy was subjected to alternate action of fatigue and corrosion in order "corrosion + fatigue in air 
TABLE 1: Chemical composition of 7B04-T6 alloy (mass fraction \%).

\begin{tabular}{lcccccccccc}
\hline Alloy & $\mathrm{Zn}$ & $\mathrm{Mg}$ & $\mathrm{Cu}$ & $\mathrm{Mn}$ & $\mathrm{Fe}$ & $\mathrm{Si}$ & $\mathrm{Ni}$ & $\mathrm{Cr}$ & $\mathrm{Ti}$ & $\mathrm{Al}$ \\
\hline 7B04-T651 & 6.23 & 2.88 & 1.58 & 0.31 & 0.15 & 0.048 & $<0.01$ & 0.16 & 0.025 & $\mathrm{Bal}$ \\
\hline
\end{tabular}

TABLE 2: Mechanical properties of the material.

\begin{tabular}{lcccc}
\hline Alloy & Direction & Yield strength, MPa & Tensile strength, MPa & Elongation \\
\hline 7B04-T651 & L-T & 573 & 497 & $11.0 \%$ \\
\hline
\end{tabular}

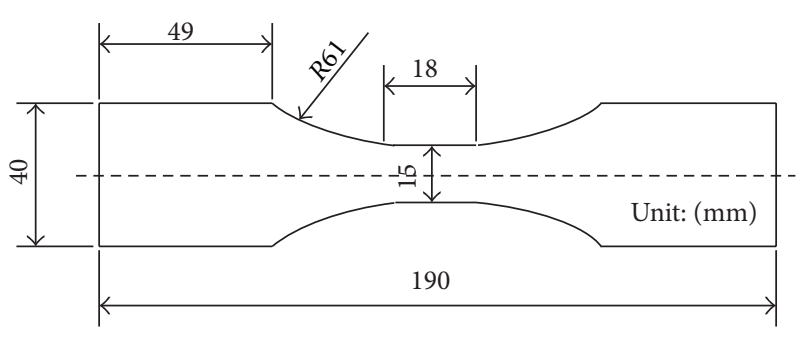

FIgURE 1: The dimension of specimens.

+ corrosion + fatigue in air $+\cdots$." The surface characteristic of specimen was shown and life variation rule was discussed in this paper. Logarithm normal and three-parameter- $(3 P$ ) Weibull distribution were applied to describe the fatigue life distribution and a better one was chosen to study the reliability fatigue life. Single side allowance factor was taken into consideration to describe the reliability fatigue life and the affection of reliability, confidence, and specimen number were discussed.

\section{Experiments}

2.1. Materials. In this paper, all the specimens are made of the 7B04-T6 aluminum alloy plate in L-T direction where the thickness of the plate is $2 \mathrm{~mm}$. The shape and size of the specimens are shown in Figure 1. In order to study the environment's affection on the plane, all specimens are covered with protection lay. The specimen in direction $S$ was polished with the emery paper in the order of \#600,\#800, and $\# 1000$.

The chemical composition and mechanical properties of 7B04-T6 aluminum alloy are shown in Tables 1 and 2, respectively.

2.2. Experiment Methodology. Completely reversed tension and tension fatigue test were conducted under a sine load waveform at constant cyclic stress frequency $(f=20 \mathrm{~Hz})$ on the MTS 810 servohydraulic test machine (MTS Systems Co., USA). The stress amplitude applied in fatigue test was $190 \mathrm{MPa}$ and stress ratio $R$ was 0.06 . The alternate load of corrosion and fatigue test specimens was divided into six sets, each with different load cycles and corrosion time, as shown in Table 3.

The solution used for the corrosion investigations was made of $3.5 \% \mathrm{NaCl}+5 \times 10^{-5} \mathrm{M} \mathrm{H}_{2} \mathrm{SO}_{4}(\mathrm{PH}=4)$ using
TABLE 3: The corroded time and fatigue cycles in each alternate progress.

\begin{tabular}{lcc}
\hline & Corroded time $(\mathrm{h})$ & Fatigue cycles \\
\hline $1-1$ & 160 & 200000 \\
$1-2$ & 320 & 150000 \\
$1-3$ & 480 & 150000 \\
$1-4$ & 640 & 150000 \\
$1-5$ & 800 & 150000 \\
$1-6$ & 960 & 100000 \\
\hline
\end{tabular}

distilled water. The spray corrosion was taken in a YWX/Q250 corrosion box. The experimental temperature was $40 \pm$ $2^{\circ} \mathrm{C}$ and the deposited salt spray was $2 \mathrm{~mL} /\left(\mathrm{h} \cdot 80 \mathrm{~cm}^{2}\right)$.

\section{Results and Discussion}

3.1. Alternate Experiment. The corrosion and fatigue test results under alternate action are listed in Table 4 . Table 5 is the total corrosion time and the alternate times of the specimens in the test. Figure 2 shows fatigues cycles and average fatigue cycles under different corrosion time. Many investigations have reported that precorrosion fatigue life data tend to show smaller dispersion than those under less precorrosion time [16-19]. However, dispersion of fatigue life data of different sets from 1-1 to 1-6 is 0.08017, 0.09886, $0.08123,0.03954,0.09188$, and 0.10089 , respectively, showing no clear trend. So the dispersion of fatigue life data for aluminum alloy under alternate action of corrosion and fatigue is much discrepant with precorrosion test.

Surface damage by corrosion and fatigue is the main failure factor of aircraft structure. Corrosion pitting has been found to be responsible for the nucleation of fatigue cracks in a wide range of aluminum alloys [20]. The examination of the specimens' surface indicated that cracks essentially nucleated and grew from corrosion pits [16]. The surface of specimens under precorrosion fatigue and corrosion fatigue always has one or two main large corrosion pits and the biggest pits are critical for pit-to-surface crack, as shown in Figure 3. However, there is much small proximity of corrosion pits growing and combined into a unit on the surface of alternate experiment specimen, as shown in Figure 3. Moreover, optical observations also prove that there are many surface splits on the alternate specimens, as shown in Figures 4(a) and 4(b).

During the alternate progress, fatigue loads produce intruding and extruding slip steps on the surface, as shown 
TABLE 4: The test data of alternate action of corrosion and fatigue experiment.

\begin{tabular}{|c|c|c|c|c|c|c|c|c|}
\hline Specimen set & Specimen number & Cycles/N & Stress level & Specimen number & Cycles/N & Stress level & Specimen number & Cycles/N \\
\hline \multirow{8}{*}{$1-1$} & 1 & 403299 & \multirow{8}{*}{$1-2$} & 1 & 201935 & \multirow{8}{*}{$1-3$} & 1 & 270594 \\
\hline & 2 & 309240 & & 2 & 216770 & & 2 & 214256 \\
\hline & 3 & 246582 & & 3 & 237487 & & 3 & 162792 \\
\hline & 4 & 247583 & & 4 & 251103 & & 4 & 270088 \\
\hline & 5 & 353761 & & 5 & 260370 & & 5 & 246267 \\
\hline & 6 & 330930 & & 6 & 276763 & & 6 & 267360 \\
\hline & 7 & 264832 & & 7 & 289039 & & 7 & 204453 \\
\hline & 8 & 338312 & & 8 & 298131 & & 8 & 193156 \\
\hline \multirow{8}{*}{$1-4$} & 1 & 240487 & \multirow{8}{*}{$1-5$} & 1 & 254722 & \multirow{8}{*}{$1-6$} & 1 & 248239 \\
\hline & 2 & 218537 & & 2 & 242083 & & 2 & 148155 \\
\hline & 3 & 225622 & & 3 & 189448 & & 3 & 256799 \\
\hline & 4 & 233794 & & 4 & 325503 & & 4 & 251726 \\
\hline & 5 & 230260 & & 5 & 161043 & & 5 & 162319 \\
\hline & 6 & 234820 & & 6 & 234710 & & 6 & 153783 \\
\hline & 7 & 273044 & & 7 & 201659 & & 7 & 175482 \\
\hline & 8 & 282169 & & 8 & 232595 & & 8 & 203641 \\
\hline
\end{tabular}

TABLE 5: The total corroded time and alternate times of the test.

\begin{tabular}{lcc}
\hline Specimen number & Alternate times & Total corrosion time (h) \\
\hline $1-1$ & 2 & 320 \\
$1-2$ & 2 & 640 \\
$1-3$ & 2 & 960 \\
$1-4$ & 2 & 1280 \\
$1-5$ & 2 & 1600 \\
$1-6$ & 2 & 1920 \\
\hline
\end{tabular}

in Figure 5. However, if the material is subjected to a corrosive environment at this stage, corrosion tends to remove intrusions and extrusions and forms many small corrosion pits and the surface roughness becomes weak and tends to form split on the surface. When the material fatigue is after corrosion again, the slips form perpendicular to stress direction. Many investigations have found that the fatigue life decreases when the corrosion time increases [15]. Figure 6 shows that specimens of 1-4 and 1-5 have longer fatigue cycles than 1-3, even though specimens of 1-4 and 1-5 are subject to longer corrosion time. When the specimens are subject to longer corrosion time, more intruding and extruding slip steps are dissolved. While the material is subject to fatigue loads again, more surface slips are formed; thus, more fatigue damage energy is dispersed. The corrosion pits of 1-4 consist of more small pits at the same size, and pits shape is not as sharp as 1-3; reducing the stress concentration leads to a longer fatigue life.

\section{Distribution Test of Fatigue Life}

Many investigations have found that logarithm normal distribution and three-parameter- $(3 P-)$ Weibull distribution were applied to describe the fatigue life data [21, 22]. In this paper, both the distributions were discussed and the best

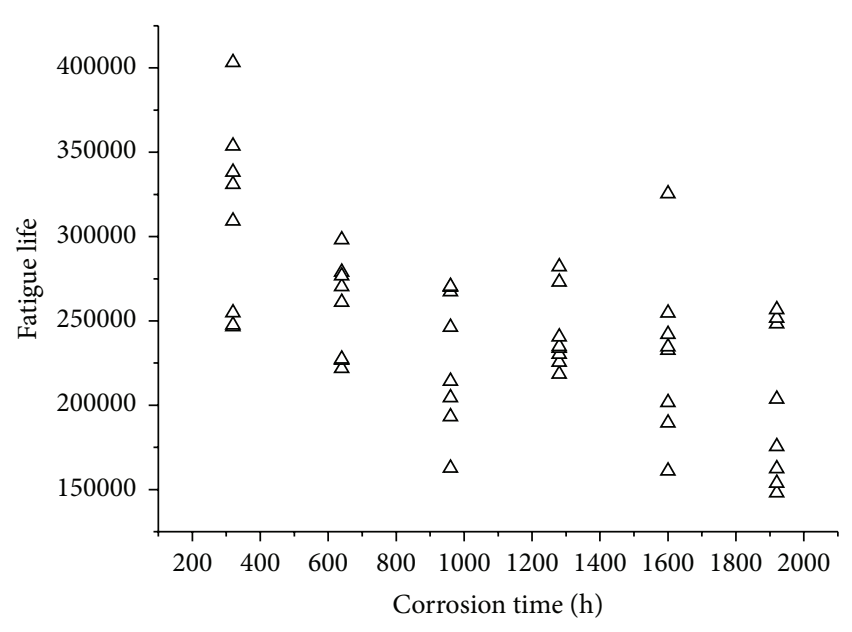

FIgURE 2: The fatigue cycles of specimens under different corrosion time.

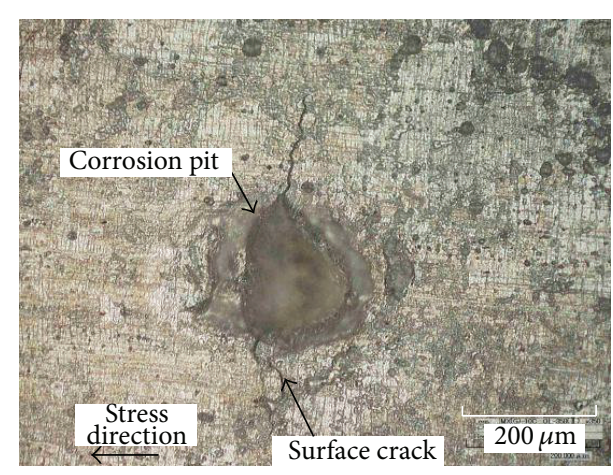

FIGURE 3: Specimen surface of precorrosion (corrosion time: $1600 \mathrm{~h})$. 


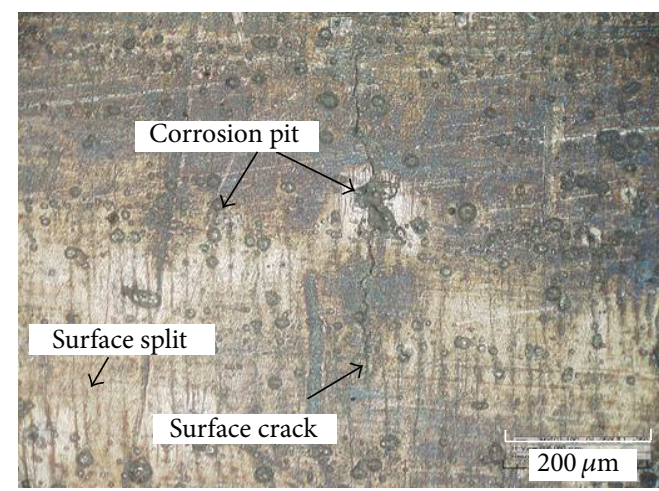

(a)

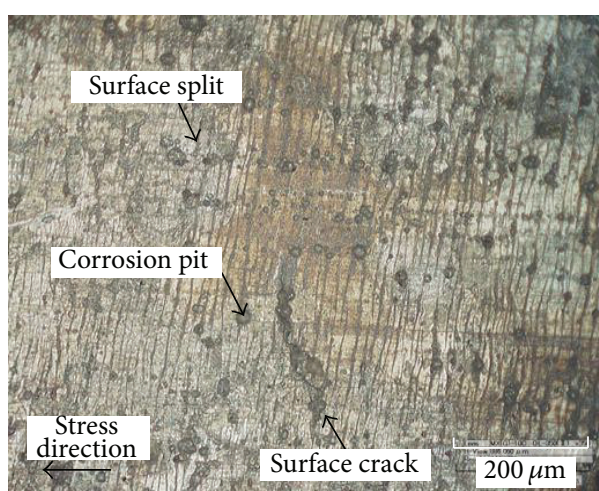

(b)

Figure 4: (a) Specimen surface of 1-3. (b) Specimen surface of 1-4.

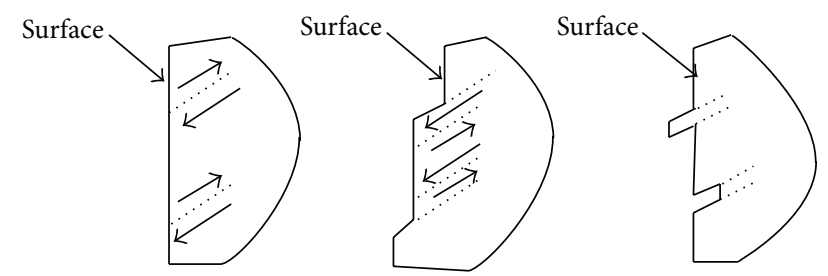

FIGURE 5: Formation of intruding and extruding slip steps under fatigue loads.

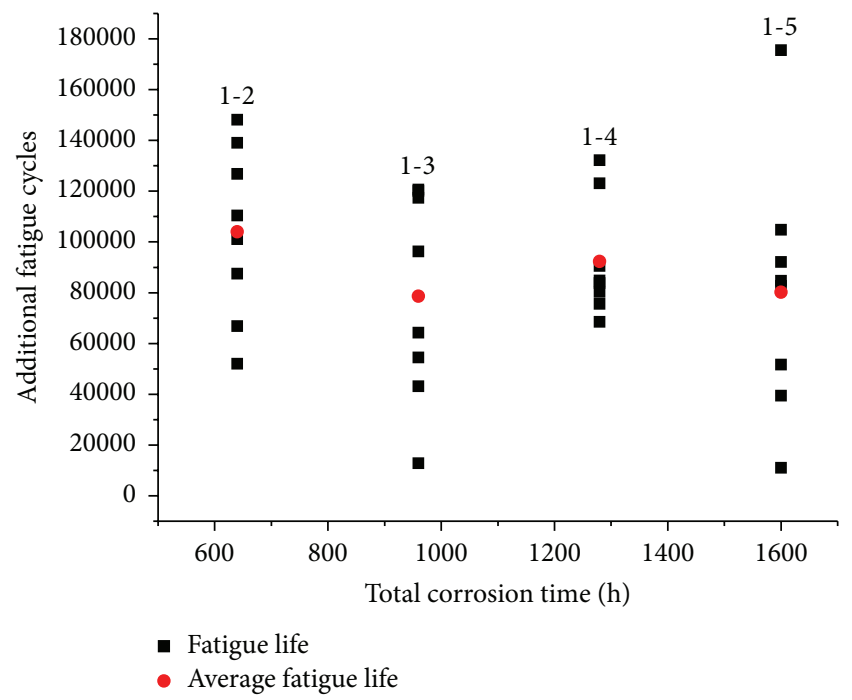

FIGURE 6: Additional fatigue cycles of specimen sets: 1-2, 1-3, 1-4, and 1-5.

fitting distribution was found out according to the correlation coefficient $r$.

4.1. Distribution of Fatigue Life. The probability density function (PDF) $f$ and cumulative distribution function (CDF) $F$ of the two distributions are expressed by following functions.
(1) Logarithm Normal Distribution. The PDF and CDF of logarithm normal distribution can be expressed by (1) and (2):

$$
\begin{aligned}
& f(\lg x)=\frac{1}{\sqrt{2 \pi} \sigma} \exp \left[-\frac{(\lg x-\mu)^{2}}{2 \sigma^{2}}\right], \\
& F(\lg x)=\int_{-\infty}^{\lg x} f(\lg x) d(\lg x)=\Phi\left[\left(\frac{\lg x-\mu}{\sigma}\right)\right] .
\end{aligned}
$$

$\lg x$ is the random variable, $\mu$ is the mathematical expectation, and $\sigma$ is the variance; (2) can be replaced by

$$
\Phi^{-1}[F(\lg x)]=-\frac{\mu}{\sigma}+\frac{1}{\sigma} \lg x .
$$

By assuming $X=\lg x, Y=\Phi^{-1}[F(\lg x)], A=-(\mu / \sigma)$, and $B=1 / \sigma$, the standard linear equation can be obtained as follows:

$$
Y=A+B X
$$

The evaluating values of $\mu, \sigma$ were calculated by using maximum likelihood estimation method in Table 4.

(2) 3P-Weibull Distribution. The PDF and CDF of 3P-Weibull distribution are expressed by

$$
\begin{aligned}
& f(x)=\frac{b}{\beta-\alpha}\left(\frac{x-\alpha}{\beta-\alpha}\right)^{b-1} \exp \left[-\left(\frac{x-\alpha}{\beta-\alpha}\right)^{b}\right] \\
& (x \geq \alpha), \\
& F(x)=1-\exp \left[-\left(\frac{x-\alpha}{\beta-\alpha}\right)^{b}\right],
\end{aligned}
$$

where $x$ is the random variable, $\alpha$ is the lower limit parameter of random variable $x$, and $b$ is the shape parameter. $\beta$ is the feature parameter which is independent of other constant parameters because $F(\beta)=1-e^{-1}$ :

$$
\left(\frac{x-\alpha}{\beta-\alpha}\right)^{b}=\ln \left(\frac{1}{1-F(x)}\right) .
$$


TABLE 6: The evaluating value of $\mu, \sigma, \alpha, \beta, b$, and $r$ for log-normal distribution and 3P-Weibull distribution.

\begin{tabular}{lccccccc}
\hline \multirow{2}{*}{ Stress level } & \multicolumn{3}{c}{ Log-normal distribution } & \multicolumn{3}{c}{$3 P$-Weibull distribution } \\
& $\mu$ & $\sigma$ & $r$ & $\alpha$ & $\beta$ & $b$ & 0.9726 \\
$1-1$ & 5.4877 & 0.07802 & 0.9742 & 53168 & 336025 & 4.1416 \\
$1-2$ & 5.4012 & 0.05967 & 0.9874 & 135467 & 272437 & 9.3821 \\
$1-3$ & 5.3527 & 0.08123 & 0.9574 & 10680 & 248776 & 4.618 & 0.976 \\
$1-4$ & 5.3828 & 0.03954 & 0.9276 & 216504 & 245162 & 0.9326 & 0.9400 \\
$1-5$ & 5.3535 & 0.09188 & 0.9749 & 110693 & 244612 & 2.4417 \\
$1-6$ & 5.2908 & 0.10089 & 0.9541 & 142996 & 210115 & 0.7841 & 0.9645 \\
\hline
\end{tabular}

Using (6) into (7), (7) can be rewritten as (8) by taking the logarithm of both sides:

$$
\ln \left[\ln \left(\frac{1}{1-F(x)}\right)\right]=-b \ln (\beta-\alpha)+b \ln (x-a) .
$$

By assuming $X=\ln (x-\alpha), Y=\ln \ln [1 /(1-F(x))]$, $A=-b \ln (\beta-\alpha)$, and $B=b$, the standard linear regression equation can be obtained as follows:

$$
Y=A+B X \text {. }
$$

The empirical frequency function is defined as follows:

$$
F\left(x_{i}\right)=\frac{i}{n+1} .
$$

$n$ is the sample size and $i$ is the serials number of fatigue life data in ascending order at the same given stress level.

The values of $\alpha, \beta$, and $b$ were calculated by using the least square method $[23,24]$ in Table 4.

4.2. Statistical Test. To determine which distribution is better to describe the fatigue life data, the correlation coefficient $r$ is applied. The correlation coefficient $r$ is defined as follows:

$$
r=\frac{L_{X Y}}{\sqrt{L_{X X} L_{Y Y}}},
$$

where

$$
\begin{aligned}
L_{X X} & =\sum_{i=1}^{n}\left(X_{i}-\bar{X}\right)^{2}, \\
L_{Y Y} & =\sum_{i=1}^{n}\left(Y_{i}-\bar{Y}\right)^{2}, \\
L_{X Y} & =\sum_{i=1}^{n}\left(X_{i}-\bar{X}\right)\left(Y_{i}-\bar{Y}\right) .
\end{aligned}
$$

There exists the equation $|r| \leq 1$. A good correlation can be affirmed between variables $X$ and $Y$ if $|r|$ is close to 1 . So the better fitting of distribution can be determined by comparing $r$ value. However, only total eight specimens are used in fatigue experiment. So it is necessary to determine a threshold value for $r$ under different sample size and confidence. The statistical test was carried out by using (13) [25]. Consider

$$
t(n-2)=\frac{r \sqrt{(n-2)}}{\sqrt{1-r^{2}}}
$$

$t(n-2)$ is Student's distribution with $n-2$ degrees of freedom

$$
r_{c}=\frac{t_{a}(n-2)}{\sqrt{(n-2)+t_{\alpha}^{2}(n-2)}} .
$$

To guarantee that the fitting is precise, $r$ should satisfy the following condition:

$$
|r| \geq r_{c}
$$

Well, $r_{c}$ is 0.621 under the sample size $n=8$ and $\alpha=0.05$.

The correlation coefficient $r$ and distribution parameter of two distributions are listed in Table 6. It is obvious that the fatigue life data fits both logarithm normal distribution and $3 P$-Weibull distribution and the correlation coefficient of both distributions is larger than 0.621 . However, $r$ of $3 P$-Weibull distribution is smaller than logarithm normal distribution, indicating that logarithm normal distribution is a better description for the fatigue data.

\section{Reliability Fatigue Life}

5.1. Single Side Allowance Factor. As mentioned above in Introduction, many investigations about fatigue life under corrosive environment focus on the scatter factor and distribution. However, confidence was not taken into consideration, especially in alternate action of corrosion and fatigue. In many fatigue experiments the specimens are not sufficient because of the high cost, so it is necessary to explore the reliability fatigue life at various reliability $p$ and confidence $\gamma$. Here, the single side allowance factor is proposed.

In previous results presented by this paper, the fatigue life data obeys logarithm normal distribution better. The logarithm reliability life $x_{p}$ with reliability $p$ can be presented as

$$
x_{p}=\mu+u_{p} \sigma .
$$

$\mu$ is the mathematical expectation, $u_{p}$ is the upper-side factor of standard normal distribution, and $\sigma$ is the standard deviation.

Because the values of $\mu$ and $\sigma$ are unknown, the logarithm reliability life $x_{p}$ can be rewritten as follows:

$$
\widehat{x}_{p}=\bar{x}+\mu_{p} \widehat{\sigma}=\bar{x}+\mu_{p} \widehat{k} s_{x} .
$$




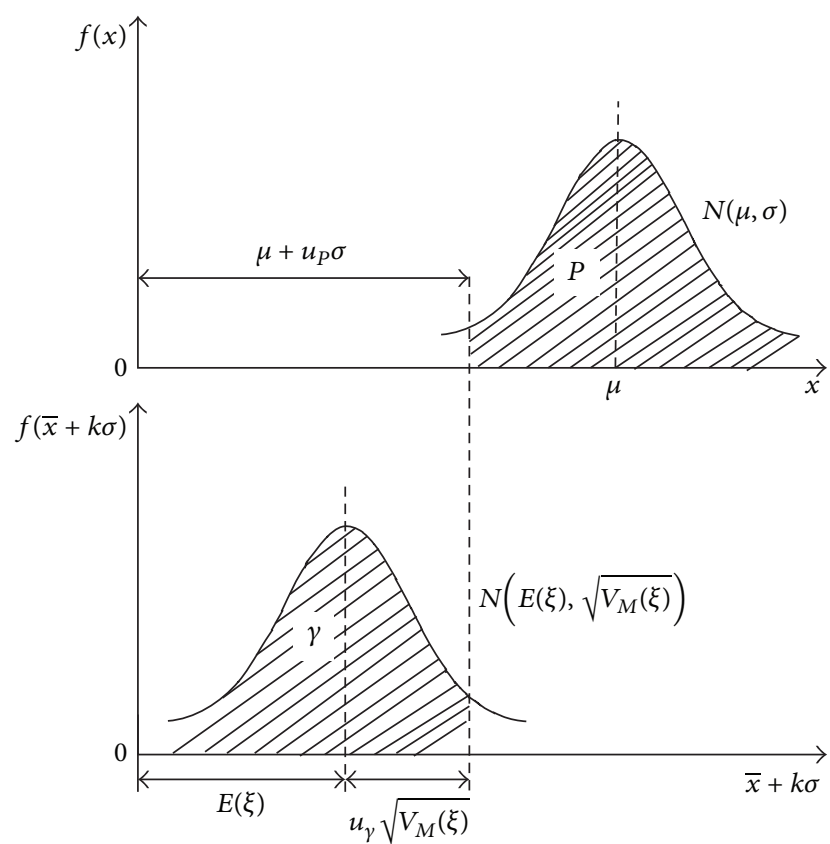

FIGURE 7: PDF of normal distribution.

$\bar{x}$ is the mean value of logarithm fatigue life data from the sample, $\widehat{\sigma}$ is the estimated value of $\sigma, s_{x}$ is the standard deviation of the sample, and $\widehat{k}$ is the correction factor of $\widehat{\sigma}$.

$\widehat{k}$ can be presented as in (18) [24-26]. Consider

$$
\widehat{k}=\sqrt{\frac{n-1}{2}} \frac{\Gamma((n-1) / 2)}{\Gamma(n / 2)} .
$$

$n$ is sample size and $\Gamma$ is gamma function.

$\mu_{p}$ in (17) can be replaced by the single side allowance factor $k$. The probability that $\bar{x}+\mu_{p} \widehat{k} \widehat{s_{x}}$ is smaller than $\mu+u_{p} \sigma$ is $\gamma$ (see Figure 7).

So

$$
P\left(\bar{x}+k \widehat{k} s_{x}<\mu+u_{p} \sigma\right)=\gamma
$$

The random variable $\xi$ is defined as

$$
\xi=\bar{x}+k \widehat{k} \widehat{s_{x}} .
$$

Assuming that $\xi$ obey the normal distribution, (16) can be expressed as (20) according to Figure 7:

$$
\mu+u_{p} \sigma=E(\xi)+\mu_{r} \sqrt{\operatorname{Var}(\xi)} .
$$

Based on the statistical theory, the mathematical expectation $E(\xi)$ is defined and calculated as

$$
E(\xi)=E(\bar{x})+k E\left(\widehat{k} s_{x}\right) .
$$

It is obvious that $E(\bar{x})=\mu, E\left(\widehat{k} s_{x}\right)=E(\widehat{\sigma})=\sigma$, so $(22)$ can be replaced by

$$
E(\xi)=\mu+k \sigma
$$

According to (20), the variance $\operatorname{Var}(\xi)$ can be written as

$$
\operatorname{Var}(\xi)=\operatorname{Var}\left(\bar{x}+k \widehat{k} s_{x}\right)=\operatorname{Var}(\bar{x})+k^{2} \widehat{k}^{2} \operatorname{Var}\left(s_{x}\right)
$$

Here

$$
\operatorname{Var}(\bar{x})=\frac{\sigma^{2}}{n}
$$

$\operatorname{Var}\left(s_{X}\right)$ is given by (26) [26]. Consider

$$
\operatorname{Var}\left(s_{X}\right)=\frac{\sigma^{2}}{n}\left\{n-1-2\left[\frac{\Gamma(n / 2)}{\Gamma((n-1) / 2)}\right]^{2}\right\} .
$$

Take (18), (25), and (26) into (24). So $\operatorname{Var}(\xi)$ can be rewritten as follows:

$$
\begin{aligned}
& \operatorname{Var}(\xi)=\frac{\sigma^{2}}{n}+k^{2}\left[\sqrt{\frac{n-1}{2}} \frac{\Gamma((n-1) / 2)}{\Gamma(n / 2)}\right]^{2} \\
& \cdot \frac{\sigma^{2}}{n-1}\left\{n-1-2\left[\frac{\Gamma(n / 2)}{\Gamma((n-1) / 2)}\right]^{2}\right\}=\frac{\sigma^{2}}{n} \\
& +k^{2}\left\{\frac{(n-1) \sigma^{2}}{2}\left[\frac{\Gamma((n-1) / 2)}{\Gamma(n / 2)}\right]^{2}-\sigma^{2}\right\} .
\end{aligned}
$$

If $n \geq 5$, (28) can be obtained as follows:

$$
\frac{(n-1)}{2}\left[\frac{\Gamma((n-1) / 2)}{\Gamma(n / 2)}\right]^{2} \approx \frac{1}{2(n-1)}+1 .
$$

Thus (27) can be simplified into

$$
\operatorname{Var}(\xi)=\sigma^{2}\left[\frac{1}{n}+\frac{k^{2}}{2(n-1)}\right] .
$$

Taking (23) and (29) into (21), (30) can be obtained as follows:

$$
\mu+u_{p} \sigma=\mu+k \sigma+u_{\gamma} \sigma \sqrt{\frac{1}{n}+\frac{k^{2}}{2(n-1)}}
$$

Then

$$
u_{p}=k+u_{r} \sqrt{\frac{1}{n}+\frac{k^{2}}{2(n-1)}}
$$

So the single side allowance factor $k$ can be presented as follows:

$$
k=\frac{u_{p}-\mu_{\gamma} \sqrt{(1 / n)\left[1-u_{\gamma}^{2} / 2(n-1)\right]+\mu_{p}^{2} / 2(n-1)}}{1-u_{\gamma}^{2} / 2(n-1)} .
$$

With given reliability $p$ and confidence $\gamma$ which can be calculated through standard normal distribution, the logreliability fatigue life $\widehat{x}_{p}$ can be calculated by

$$
\widehat{x}_{p}=\bar{x}+k \widehat{\sigma} .
$$




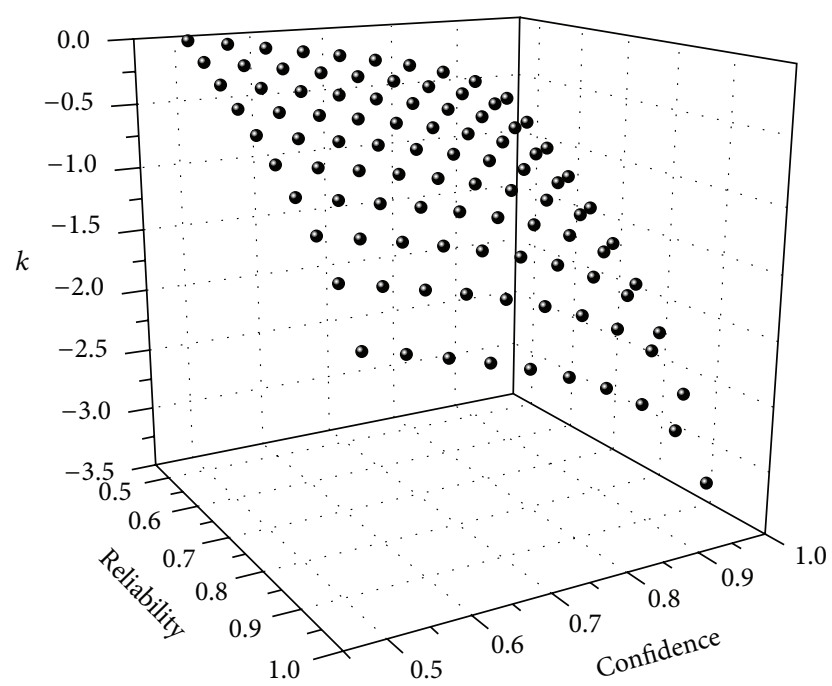

Figure 8: Single side allowance factor $k(n=8)$.

Finally the reliability fatigue life $N_{p, \gamma}$ with reliability $p$ and confidence $\gamma$ can be calculated by

$$
N_{p, \gamma}=10^{\widehat{x}_{p}}
$$

The value of $k$ with various reliability from $50 \%$ to $95 \%$ (increasing in step of 5\%), and confidence from 50\% to $95 \%$ (increasing in step of 5\%) was calculated based on (32). As shown in Figure $8(n=8)$, the value of $k$ decreases when reliability $p$ and confidence $\gamma$ increase at the same $n$ state.

The value of $k$ tends to increase slowly and reach a stable constant with specimens number $n$ increasing, as shown in Figures 9 and 10 (especially $p=50 \%$, the value of $k$ is constant, $k=-1.6499) . n=8$ is the critical point of increasing velocity of $k$; when $n<8$, the value of $k$ rises rapidly with the increasing of specimen number $n$. While $n>9, k$ rises smoothly with the increasing of $n$. So in order to achieve a higher reliability and stable fatigue life with a given reliability and confidence more specimens are needed and the amount should be larger than eight.

5.2. Reliability Fatigue Life. Reliability fatigue life $N_{p, \gamma}$ with reliability $p=95 \%$ and confidence $\gamma=50 \%, 75 \%, 85 \%$, and $95 \%$ was listed in Table 7 . Reliability fatigue life increases with the confidence decreasing. $N_{p, \gamma}$ at confidence $\gamma=95 \%$ and reliability $p=50 \%, 75 \%, 85 \%$, and $95 \%$ was calculated and shown in Table 8. Safe fatigue life of a structure is that number of events such as flights, landings, or flight hours, during which there is a low probability that the strength will degrade below its design ultimate value due to fatigue cracking [27]. The safe fatigue life is one main and important index for administrating aircraft service life and ensuring safety which is determined by fatigue test. When the test data obeys logarithm normal distribution, the safe fatigue life is fatigue cycles at $99.9 \%$ reliability and $90 \%$ confidence [2]. So the safe fatigue life is $N_{p, \gamma}$ at $99.9 \%$ reliability and $90 \%$ confidence, listed in Table 9.

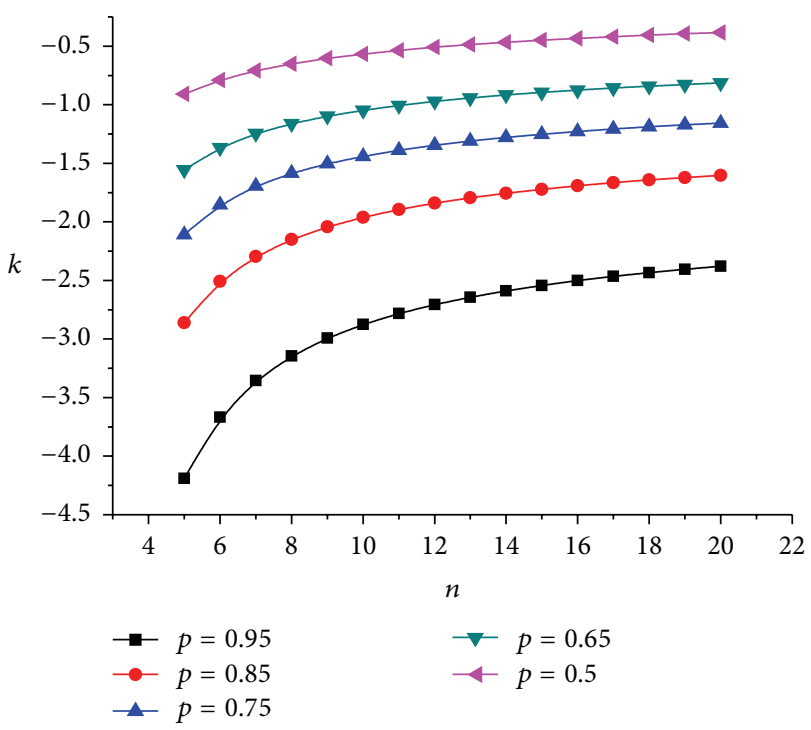

FIGURE 9: Single side allowance factor $k$ versus $n$ at various reliability $(\gamma=95 \%)$.

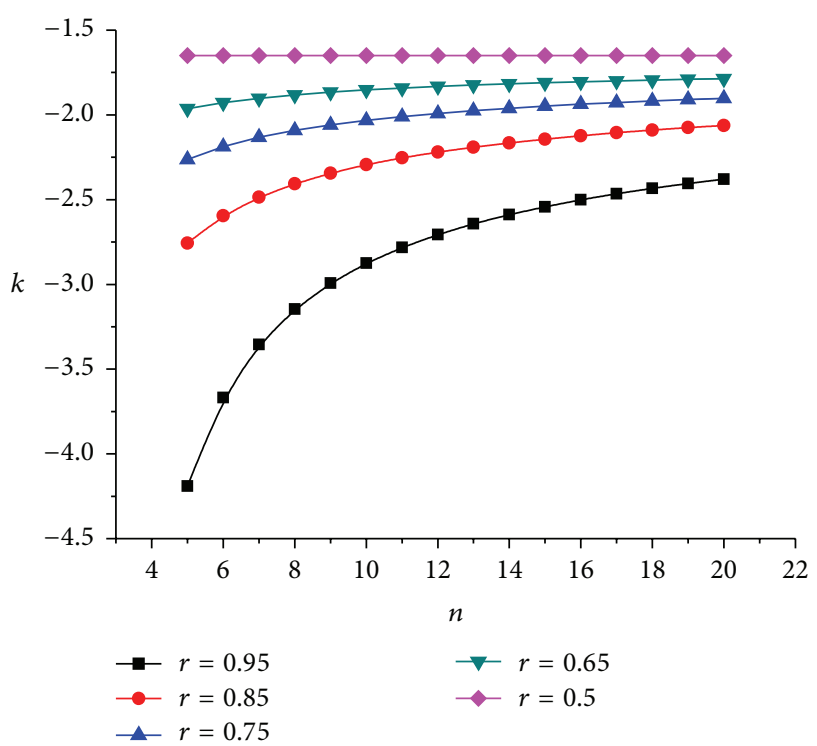

FIgURE 10: Single side allowance factor $k$ versus $n$ at various confidence $(p=95 \%)$.

The median fatigue life has larger scale than safe fatigue life in each set, as shown in Figure 11. It indicates that it is safe to administrate the aircraft life using safe fatigue life. Figure 11 also shows that the fatigue life of the same set at reliability $p=$ 0.95 and confidence $\gamma=0.85$ is longer than that at reliability $p=0.85$ and confidence $\gamma=0.95$. So the reliability has more serious effect on the value of fatigue life than confidence.

\section{Conclusion}

Alternate action of corrosion and fatigue experimentation of AA7B04 was carried out to investigate the fatigue performances. The different characteristics between precorrosion 
TABLE 7: Reliability fatigue life $N_{p, \gamma}$ under various confidence ( $\left.p=95 \%\right)$.

\begin{tabular}{|c|c|c|c|c|c|c|c|c|}
\hline \multirow{3}{*}{ Stress level } & \multicolumn{8}{|c|}{ Reliability 0.95} \\
\hline & \multicolumn{2}{|c|}{ Confidence 0.5} & \multicolumn{2}{|c|}{ Confidence 0.75} & \multicolumn{2}{|c|}{ Confidence 0.85} & \multicolumn{2}{|c|}{ Confidence 0.95} \\
\hline & $\operatorname{Lg} N_{p, \gamma}$ & $N_{p, \gamma}$ & $\operatorname{Lg} N_{p, \gamma}$ & $N_{p, \gamma}$ & $\operatorname{Lg} N_{p, \gamma}$ & $N_{p, \gamma}$ & $\operatorname{Lg} N_{p, \gamma}$ & $N_{p, \gamma}$ \\
\hline $1-1$ & 5.3594 & 228770 & 5.3246 & 211154 & 5.3000 & 199526 & 5.2424 & 174743 \\
\hline $1-2$ & 5.3030 & 200909 & 5.2764 & 188973 & 5.2577 & 181009 & 5.2136 & 163531 \\
\hline $1-3$ & 5.2191 & 165615 & 5.1829 & 152370 & 5.1573 & 143648 & 5.0973 & 125112 \\
\hline $1-4$ & 5.3178 & 207874 & 5.3001 & 199572 & 5.2877 & 193955 & 5.2585 & 181343 \\
\hline $1-5$ & 5.2024 & 159368 & 5.1614 & 145011 & 5.1325 & 135675 & 5.0646 & 116038 \\
\hline $1-6$ & 5.1248 & 133291 & 5.0798 & 120171 & 5.0481 & 111712 & 4.9735 & 94081 \\
\hline
\end{tabular}

TABLE 8: Reliability fatigue life $N_{p, \gamma}$ under various reliability $(\gamma=95 \%)$.

\begin{tabular}{|c|c|c|c|c|c|c|c|c|}
\hline \multirow{3}{*}{ Stress level } & \multicolumn{8}{|c|}{ Confidence 0.95} \\
\hline & \multicolumn{2}{|c|}{ Reliability 0.5} & \multicolumn{2}{|c|}{ Reliability 0.75} & \multicolumn{2}{|c|}{ Reliability 0.85} & \multicolumn{2}{|c|}{ Reliability 0.95} \\
\hline & $\operatorname{Lg} N_{p, \gamma}$ & $N_{p, \gamma}$ & $\operatorname{Lg} N_{p, \gamma}$ & $N_{p, \gamma}$ & $\operatorname{Lg} N_{p, \gamma}$ & $N_{p, \gamma}$ & $\operatorname{Lg} N_{p, \gamma}$ & $N_{p, \gamma}$ \\
\hline $1-1$ & 5.4372 & 273653 & 5.3644 & 231420 & 5.3204 & 209122 & 5.2424 & 174743 \\
\hline $1-2$ & 5.3626 & 230462 & 5.3069 & 202722 & 5.2733 & 187629 & 5.2136 & 163531 \\
\hline $1-3$ & 5.3001 & 199572 & 5.2243 & 167610 & 5.1786 & 150869 & 5.0973 & 125112 \\
\hline $1-4$ & 5.3572 & 227615 & 5.3203 & 209074 & 5.2980 & 198609 & 5.2585 & 181343 \\
\hline $1-5$ & 5.2940 & 196789 & 5.2083 & 161547 & 5.1565 & 143384 & 5.0646 & 116038 \\
\hline $1-6$ & 5.2255 & 168074 & 5.1313 & 135301 & 5.0745 & 118713 & 4.9735 & 94081 \\
\hline
\end{tabular}

TABlE 9: Safe fatigue life of different specimen set.

\begin{tabular}{lc}
\hline Specimen set & Safe fatigue life \\
\hline $1-1$ & 129927 \\
$1-2$ & 130377 \\
$1-3$ & 91897 \\
$1-4$ & 156063 \\
$1-5$ & 81865 \\
$1-6$ & 64150 \\
\hline
\end{tabular}

and alternate action of corrosion and fatigue surface were studied. Special attention was given to the reliability fatigue life of using single side allowance factor. The major results and conclusions are summarized as follows.

(1) The fatigue life of alternate experimentation does not always decrease with the increasing of total corrosion time as the precorrosion test. The dispersion of fatigue under alternate experiment has obvious rule as precorrosion. No big-main corrosion pit and surface slips are distinct characteristic of alternate experiment.

(2) Fatigue life data of alternate experiment follows both logarithm normal distribution and $3 P$-Weibull distribution but logarithm normal distribution has a better agreement.

(3) One-side allowance factor $k$ is proposed to predict the reliability fatigue life $N_{p, \gamma}$ based on logarithm normal distribution. The value of $k$ increases with the decreasing of reliability and confidence.

The specimen number also had affection on the one-side allowance factor. The one-side allowance factor $k$ increases

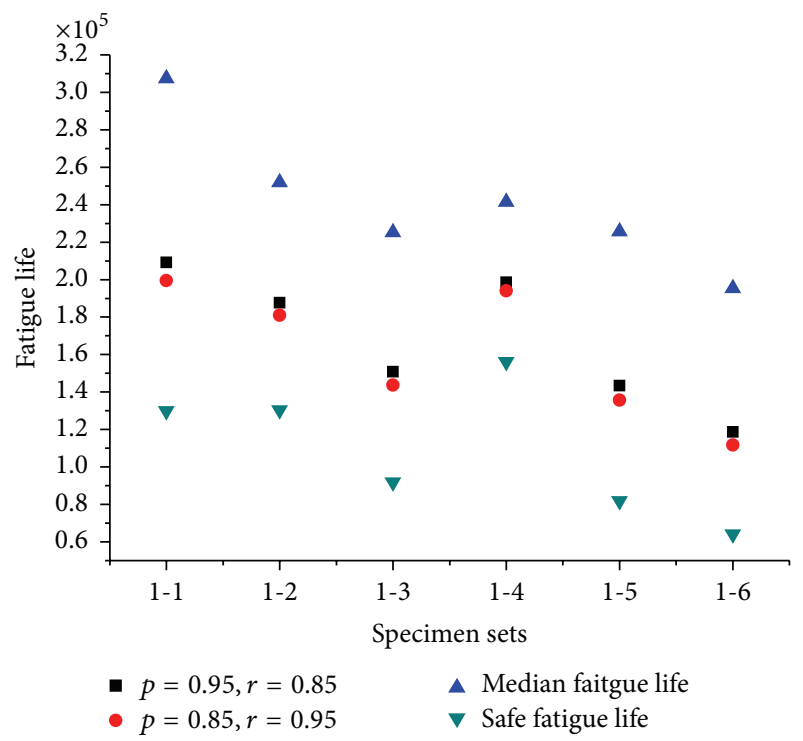

FIGURE 11: Reliability fatigue life and median fatigue life of different specimen set at various reliability and confidence.

with increasing of specimen number and $n=8$ is the critical point of increasing velocity.

(4) Safe fatigue life is smaller than median fatigue life and it is safe to administrate aircraft life using safe fatigue life.

\section{Competing Interests}

The authors declare that they have no competing interests. 


\section{Acknowledgments}

The authors are grateful to financial support for this study granted by National Natural Science Foundation of China (51475470) and to AVIC Beijing Institute of Aeronautics Materials, where all the specimens were manufactured.

\section{References}

[1] Y. Kim, S. Sheehy, and D. Lenhardt, A Survey of Aircraft Structural-Life Management Programs in the U.S. Navy, the Canadian Forces, and the U.S. Air Force, RAND, Santa Monica, Calif, USA, 2006.

[2] Z. Jiang, D. Tian, and Z. Zhou, Fatigue Loads and Environment Spectrum of Aircraft Structure, Publishing House of Electronics Industry, 2012.

[3] T. H. Flournoy and C. C. Seher, Corrosion Activity in the Federal Aviation Administration's National Aging Aircraft Research Program, Corrosion 95, Orlando, Fla, USA, 1995.

[4] S. Ishihara, S. Saka, Z. Y. Nan, T. Goshima, and S. Sunada, "Prediction of corrosion fatigue lives of aluminium alloy on the basis of corrosion pit growth law," Fatigue and Fracture of Engineering Materials and Structures, vol. 29, no. 6, pp. 472-480, 2006.

[5] H. Xiao-Guang and X. Jin-Quan, "Pit morphology characterization and corrosion fatigue crack nucleation analysis based on energy principle," Fatigue and Fracture of Engineering Materials and Structures, vol. 35, no. 7, pp. 606-613, 2012.

[6] K. K. Sankaran, R. Perez, and K. V. Jata, "Effects of pitting corrosion on the fatigue behavior of aluminum alloy 7075T6: modeling and experimental studies," Materials Science and Engineering A, vol. 297, no. 1-2, pp. 223-229, 2001.

[7] K. Jones and D. W. Hoeppner, "Prior corrosion and fatigue of 2024-T3 aluminum alloy," Corrosion Science, vol. 48, no. 10, pp. 3109-3122, 2006.

[8] T. C. Lindley, P. Mcintyre, and P. J. Trant, "Fatigue-crack initiation at corrosion pits," Metals Technology, vol. 9, no. 1, pp. 135$142,1982$.

[9] Y. Kondo, "Prediction of fatigue crack initiation life based on pit growth," Corrosion, vol. 45, no. 1, pp. 7-11, 1989.

[10] M. L. Du, F. P. Chiang, S. V. Kagwade, and C. R. Clayton, "Damage of Al 2024 alloy due to sequential exposure to fatigue, corrosion and fatigue," International Journal of Fatigue, vol. 20, no. 10, pp. 743-748, 1998.

[11] F. Menan and G. Henaff, "Influence of frequency and exposure to a saline solution on the corrosion fatigue crack growth behavior of the aluminum alloy 2024," International Journal of Fatigue, vol. 31, no. 11-12, pp. 1684-1695, 2009.

[12] F. Menan and G. Henaff, "Synergistic action of fatigue and corrosion during crack growth in the 2024 aluminum alloy," Pocedia Fngrneering, vol. 2, no. 3, pp. 1441-1450, 2010.

[13] Y. L. Chen, G. X. Bian, L. Yi, and Y. Zhang, "Research on fatigue characteristic and fracture mechanics of aluminum alloy under alternate action of corrosion and fatigue," Journal of Mechanical Engineering, vol. 48, no. 20, pp. 70-76, 2012.

[14] P. Jin and X. H. Yang, "Fatigue damage competitive rule after corrosive environment action," Chinese Journal of Applied Mechanics, vol. 21, no. 3, pp. 72-75, 2004.

[15] C. H. Fan, Study on Some Critical Problem for Single Aircraft Structure Life Surveillance, Air Force Engineering University, 2010.
[16] K. Jones, Effect of microstructure on pit-to-crack transition of 7075-T6 Aluminum Alloy [Ph.D. dissertation], University of Utah, 2004.

[17] D. W. Hoeppner, "Model for prediction of fatigue lives based upon a pitting corrosion fatigue process," ASTM STP 675, American Society for Testing and Materials, 1979.

[18] R. M. Pidaparti, M. J. Palakal, and L. Fang, "Cellular automation approach to model aircraft corrosion pit damage growth," AIAA Journal, vol. 42, no. 12, pp. 2562-2569, 2004.

[19] Y. Chen, X. Yang, and H. Qin, "Study on corrosion damage distribution law of aircraft structure," Materials Science \& Engineering, vol. 20, no. 3, pp. 376-381, 2002.

[20] G. H. Bray, R. J. Bucci, E. L. Colvin, and M. Kulak, "Effects of prior corrosion on the $\mathrm{S} / \mathrm{N}$ fatigue performance of aluminum alloys 2024-T3 and 2524-T3," in Effects of Environment on the Initiation of Crack Growth ASTM STP 1298, vol. 89, ASTM, Philadelphia, Pa, USA, 1997.

[21] C. L. Liu, Z. Z. Lu, Y. L. Xu, and Z. F. Yue, "Reliability analysis for low cycle fatigue life of the aeronautical engine turbine disc structure under random environment," Materials Science and Engineering A, vol. 395, no. 1-2, pp. 218-225, 2005.

[22] S. Sadek and M. Olsson, "New models for prediction of high cycle fatigue failure based on highly loaded regions," International Journal of Fatigue, vol. 66, pp. 101-110, 2014.

[23] C. Chen, Fatigue and Fracture, Huazhong Science and Technology University Press, 2002.

[24] S. Qin and W. Cui, "Effect of corrosion models on the timedependent reliability of steel plated elements," Marine Structures, vol. 16, no. 1, pp. 15-34, 2003.

[25] H. O. Fuchs and R. I. Stephens, Metal Fatigue in Engineering, John Wiley \& Sons, New York, NY, USA, 1980.

[26] Z. Gao, Fatigue Applied Statistics, National Defence Industry Press, 1986.

[27] United States Department of Defense, Aircraft Structural Integrity Program, MIL-STD-1530C, USAF, Washington, DC, USA, 2005 


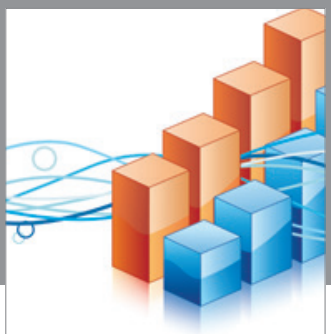

Advances in

Operations Research

vatem alat4

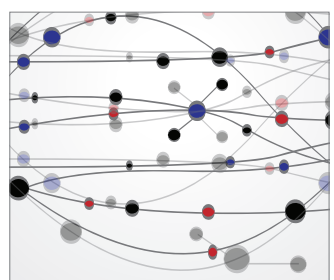

\section{The Scientific} World Journal
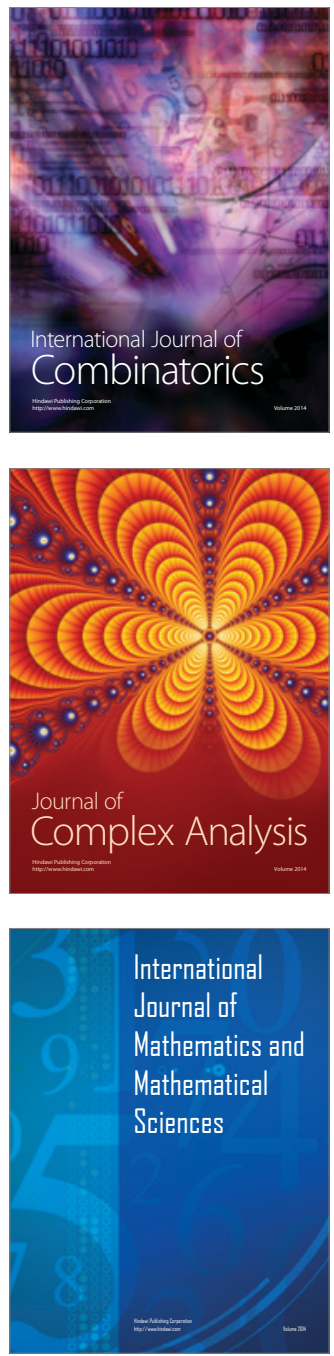
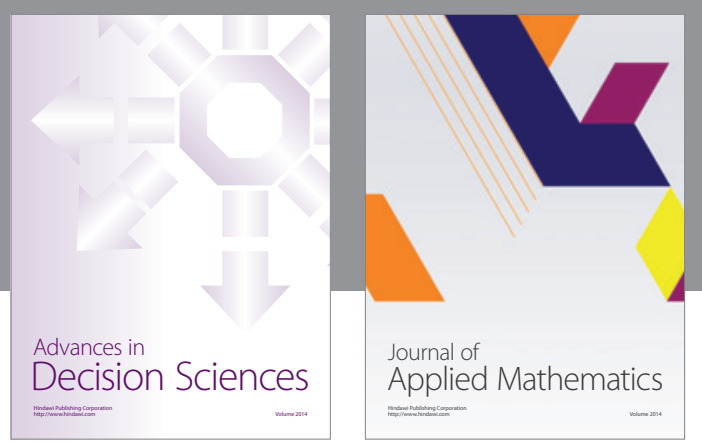

Algebra

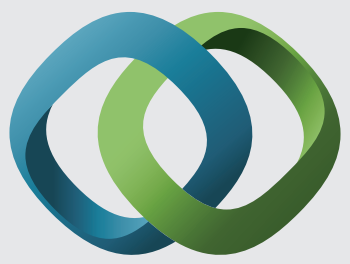

\section{Hindawi}

Submit your manuscripts at

http://www.hindawi.com
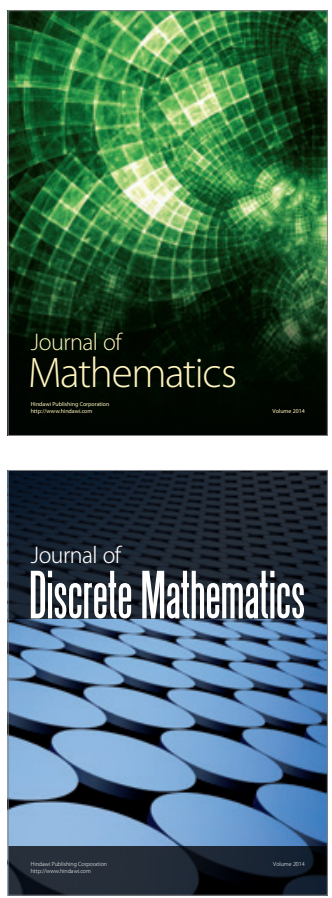

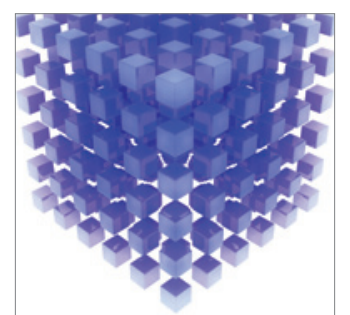

Mathematical Problems in Engineering
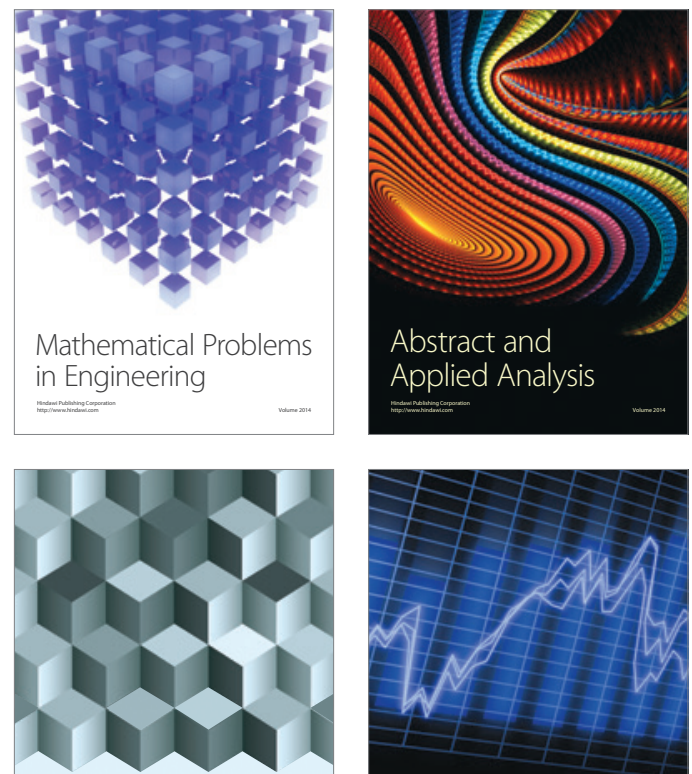

Journal of

Function Spaces

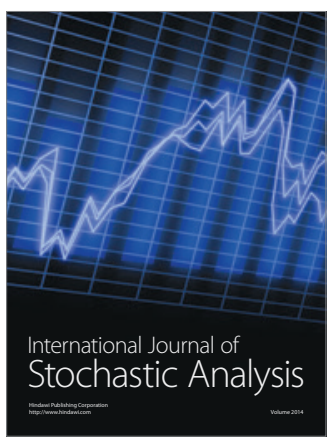

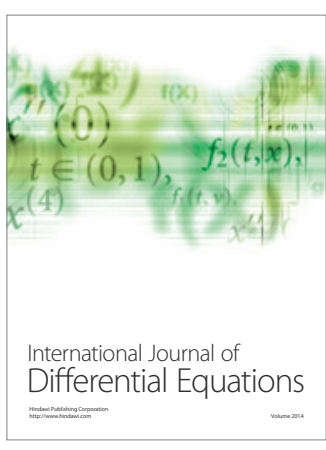
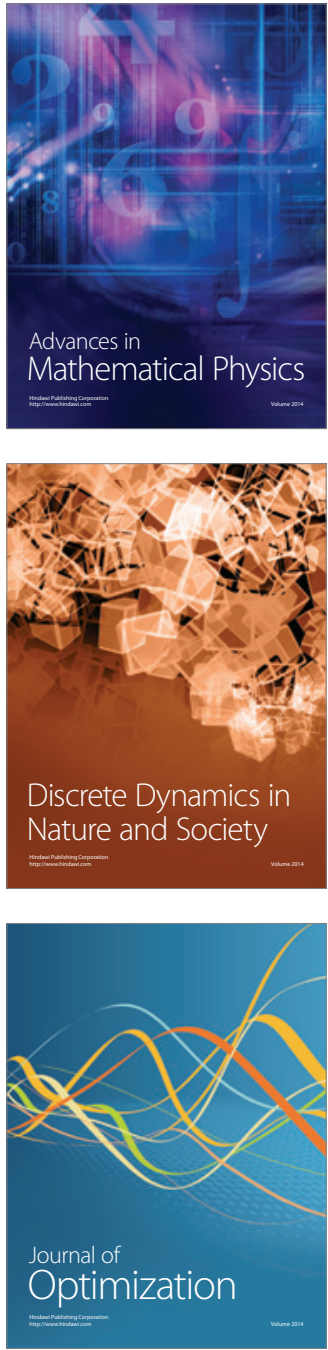